\title{
FLUID DYNAMIC COMPARISON OF INTRA-ATRIAL AND EXTRACARDIAC TOTAL CAVOPULMONARY CONNECTIONS
}

Albert C. Lardo, $\mathrm{PhD}^{\mathrm{a}}$

Steven A. Webber, MD

Ingeborg Friehs, $\mathrm{MD}^{\mathrm{b}}$

Pedro J. del Nido, MD $^{\mathrm{b}}$

Edward G. Cape, $\mathrm{PhD}^{\mathrm{a}}$
Objective: Extracardiac total cavopulmonary connection has recently been introduced as an alternative to intra-atrial procedures. The purpose of this study was to compare the hydrodynamic efficiency of extracardiac and intra-atrial lateral tunnel procedures in total cavopulmonary connections. Methods: Intra-atrial lateral tunnel, extracardiac tunnel, and extracardiac conduit with and without caval vein offset were performed on explanted sheep heart preparations and studied in an in vitro flow loop. A rate of fluid-energy dissipation analysis was performed for each model using simultaneous measurement of pressure and flow at each inlet and outlet of the right side of the heart. Preparations were perfused by using a steady flow blood pump at 4 flow indices $\left(1-6 \mathrm{~L} / \mathrm{min} / \mathrm{m}^{2}\right)$ with the inferior vena cava carrying $65 \%$ of the total venous return. Results: Fluid-power losses were consistently lower for the extracardiac conduit procedure compared with the two tunnel configurations $(P<.01)$. A further reduction in energy dissipation of up to $36 \%$ was noted in the extracardiac procedure, with $5 \mathrm{~mm}$ offset of the extracardiac conduit toward the distal right pulmonary. The intra-atrial and extracardiac tunnel procedures were least efficient, with losses $73 \%$ greater than the optimal extracardiac conduit procedure. Conclusions: The extracardiac conduit procedure provides superior hemodynamics compared with the intra-atrial lateral tunnel and extracardiac tunnel techniques. This hydrodynamic advantage is markedly enhanced by the use of conduit-superior vena cava offset, particularly at high physiologic flows that are representative of exercise. These data suggest additional rationale for the use of extracardiac conduit procedures for finalstage completion of the Fontan circulation. (J Thorac Cardiovasc Surg 1999;117:697-704)
T he intracardiac lateral tunnel total cavopulmonary connection (TCPC) has experienced widespread use over the past 10 years for the treatment of patients with complex univentricular heart disease. ${ }^{1}$ The great enthusiasm for the use of this procedure was driven by the contention that elimination of the right atrial chamber

From the Cardiac Dynamics Laboratory, ${ }^{a}$ Division of Cardiology, Children's Hospital of Pittsburgh, University of Pittsburgh, Pittsburgh, $\mathrm{Pa}$, and Department of Cardiac Surgery, ${ }^{\mathrm{b}}$ Boston Children's Hospital, Harvard University, Boston, Mass.

Received for publication June 23, 1998; revisions requested Sept 17, 1998; revisions received Oct 30, 1998; accepted for publication Nov 30, 1998.

Address for reprints: Albert C. Lardo, PhD, Johns Hopkins University School of Medicine, 407 Traylor Building, 720 Rutland Ave, Baltimore, MD 21205.

Copyright (C) 1999 by Mosby, Inc.

$0022-5223 / 99 \$ 8.00+0 \quad \mathbf{1 2 / 1 / 9 6 2 0 3}$ would result in improved hemodynamic efficiency and might also reduce the incidence of right atrium-related complications encountered in the atriopulmonary connection (supraventricular arrhythmias and atrial thrombus formation). Although TCPC may reduce the incidence of these complications in the early postoperative course ${ }^{2}$ this issue is controversial,${ }^{3}$ and moderate to late outcome remains complicated in many patients by rhythm disturbances, thrombus formation, low-output states, protein-losing enteropathy, and chronic effusions. ${ }^{4-6}$ Since some of these complications are related to extensive intra-atrial suture lines and the placement of intracardiac prosthetic material, exploration of alternative means for achieving the TCPC circulation is warranted.

Extracardiac procedures have received recent attention because they are technically simpler, do not require intra-atrial suture lines, and eliminate potentially 


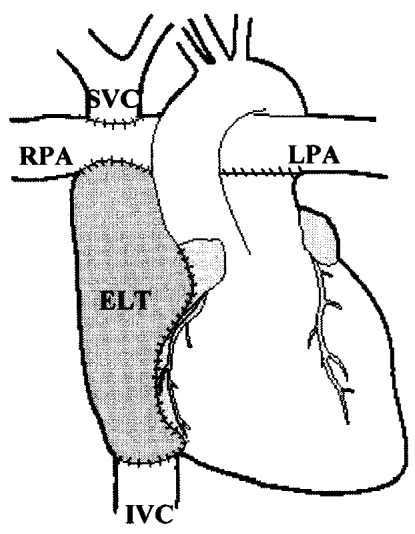

a)

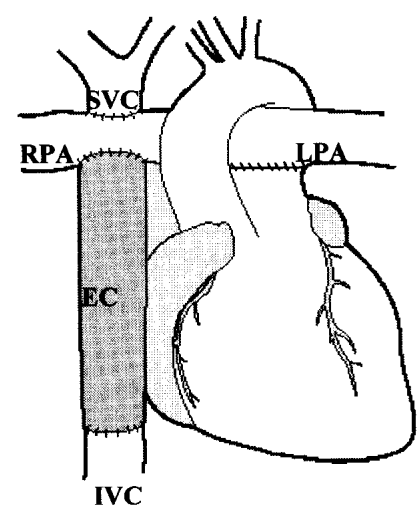

b)

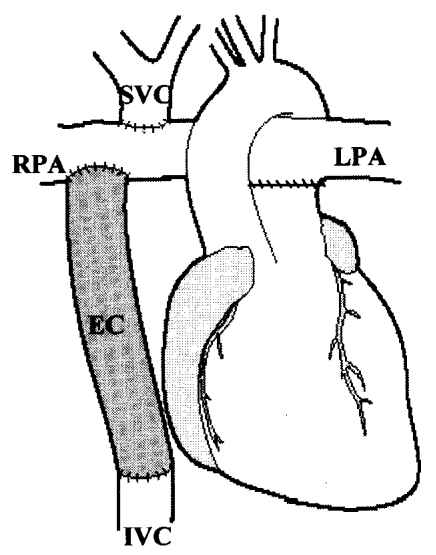

c)

Fig 1. Diagram of extracardiac procedures: a, extracardiac tunnel; b, extracardiac conduit; and c, extracardiac conduit with caval offset.

thrombogenic intra-atrial material. Within the extracardiac approach, an epicardial tunnel or a conduit can be used to divert inferior vena cava flow to the pulmonary arteries. The extracardiac conduit approach has several important theoretical advantages, including simplicity of offsetting superior and inferior vena cava flow streams, which has been shown to improve hydrodynamic efficiency. ${ }^{7-10}$ Subtle geometric differences between the extracardiac procedures and the commonly used intra-atrial lateral tunnel TCPC may have significant implications for the fluid dynamics and hydrodynamic efficiency of the surgical connection. Whereas previous researchers have described the clinical advantages of extracardiac procedures, a quantitative fluiddynamic assessment of this approach has not been previously performed. The purpose of this study was to compare the fluid dynamics of the extracardiac tunnel and conduit procedures to the intra-atrial TCPC and establish an independent fluid mechanical rationale for the use of one or more of these procedures.

\section{Methods}

Extracardiac and intra-atrial TCPC were performed on fresh explanted sheep heart preparations (subject weight, 45 $\mathrm{kg}$; body surface area, $1.2 \mathrm{~m}^{2}$ ) and studied in a previously described in vitro flow loop. ${ }^{11}$ Three procedures were studied: (1) intra-atrial lateral tunnel, (2) extracardiac epicardial tunnel, and (3) extracardiac conduit with and without $5 \mathrm{~mm}$ conduit offset toward the distal right pulmonary artery. This offset was selected on the basis of previous caval offset optimization studies on the total cavopulmonary anastomosis performed in our laboratory. ${ }^{10}$ To determine the effect of the extracardiac conduit to inferior vena cava diameter ratio, we tested 2 diameter ratios (1.0 and 1.5) to simulate a uniform extracardiac conduit-inferior vena cava transition and an oversized conduit as would be required in younger children to allow for vessel growth, respectively. The pressure in each inlet and outlet vessel was measured by using side-hole fluidfilled catheters inserted through the respective vessel cannula. The total flow rate was measured by using a calibrated rotometer, and the flows in the inferior vena cava and the right pulmonary artery were determined with ultrasound flow probes placed loosely around each vessel wall (Transonic Systems, Utica, NY). For the approximation of the normal pulmonary vascular resistance, distal pulmonary artery resistances were set to achieve a 55\%/45\% pulmonary artery flow split, favoring the right pulmonary artery, and held constant throughout the study. Explanted preparations were perfused by means of a steady-flow pump at four flow indices (1-6 $\mathrm{L} / \mathrm{min} / \mathrm{m}^{2}$ ), with the inferior vena cava carrying $65 \%$ of the total venous return. The Reynolds number, which is a dimensionless quantity that characterizes the ratio of inertial to viscous forces in a flow field, ranged from approximately 200 to 3000 in the caval veins and pulmonary arteries. A rate of fluid-energy loss (power) analysis was performed for each model by using simultaneous measurement of pressure, flow, and velocity at each inlet and outlet of the right side of the heart, as described previously. ${ }^{11}$

\section{Flow models and surgical procedures}

Intra-atrial lateral tunnel. The intra-atrial lateral tunnel TCPC was constructed by the method described by de Leval and colleagues. ${ }^{1}$ Briefly, the superior vena cava was transected and its proximal end connected directly to the superior aspect of the right pulmonary artery. The right atrium was then opened with an oblique incision, and an appropriately sized semicircular polytetrafluoroethylene tunnel was sutured into the right atrium such that the lateral wall of the atrium represented approximately one third of the total flow area. 


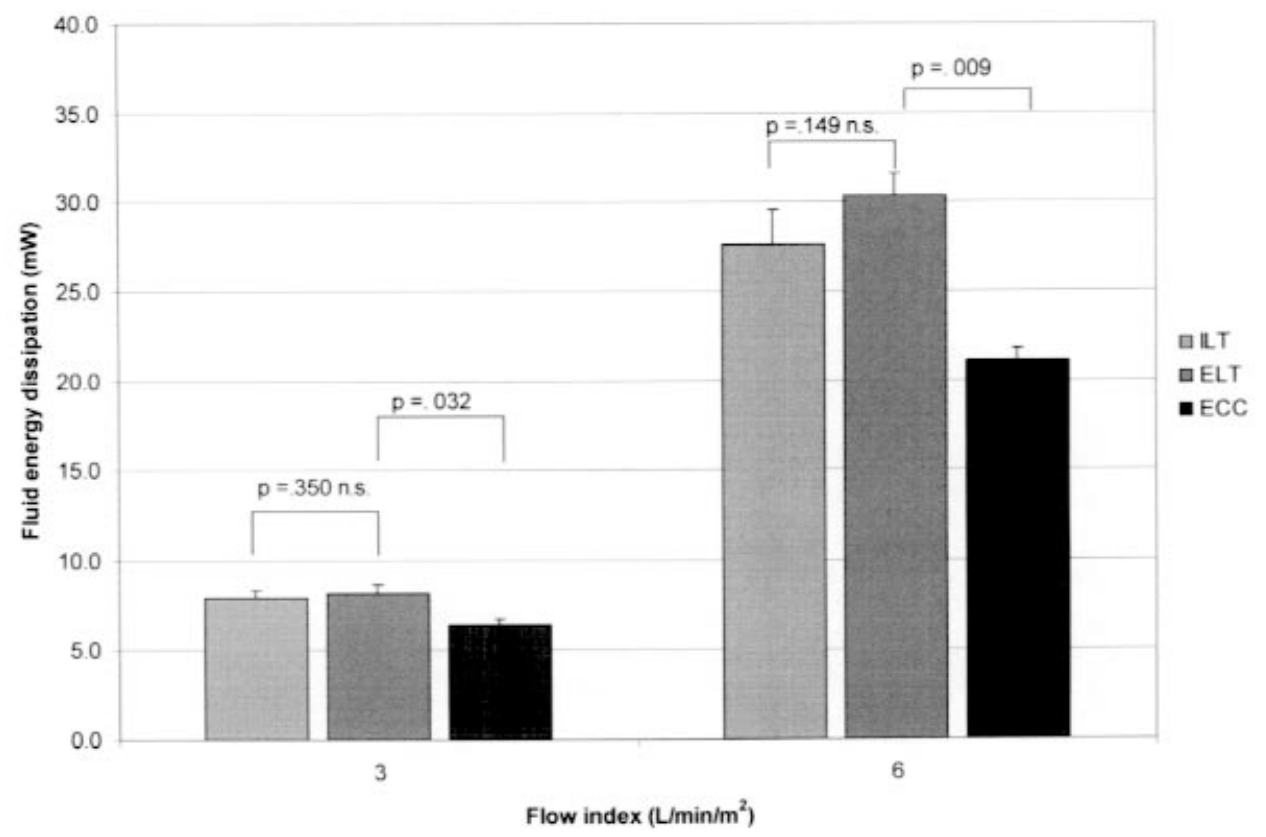

Fig 2. Fluid-power loss comparison of the intracardiac, extracardiac tunnel, and extracardiac conduit procedures at two flow indices (ILT, intracardiac lateral tunnel; ELT, extracardiac lateral tunnel; ECC, extracardiac conduit).

The distal end of the transected superior vena cava was then connected to the inferior aspect of the right pulmonary artery with the caval veins aligned $(n=6)$.

Extracardiac lateral tunnel. The extracardiac lateral tunnel procedure is shown in Fig 1, $a$. After the creation of an endto-side bidirectional superior cavopulmonary anastomosis, the atrial end of the superior vena cava was oversewn and the inferior aspect of the right pulmonary artery was incised to create an appropriately sized opening. The posterior border of the opening was then anastomosed to the epicardial surface of the atrium at the level of the atrial appendage. The inferior vena cava was then divided close to the atrium, oversewn on the atrial side, and the posteromedial border of the inferior vena cava was attached to the anterolateral epicardial atrial surface. Finally, a polytetrafluoroethylene baffle was sutured to the anterior borders of the inferior and superior venae cavae and the lateral epicardial surface of the right atrium to divert inferior vena cava blood into the proximal right pulmonary artery. Thus, the lumen cross-section for flow consists of the epicardial surface of the right atrium and the anterolateral portion of the prosthetic tunnel. Suture lines are largely epicardial, although some full-thickness bites were necessary $(n=6)$.

Extracardiac conduit. A schematic of the extracardiac conduit procedure is shown in Fig 1, b. As described for the intraatrial procedure, venous return from the upper extremities was diverted to the pulmonary arteries via a superior vena cava to superior right pulmonary artery end-to-side anastomosis. After the division of the inferior vena cava $2 \mathrm{~cm}$ proximal to its entrance to the right atrium, lower extremity venous return was diverted to the pulmonary arteries via a polytetrafluoroethylene conduit of uniform cross-section sutured end to side to the inferior aspect of the proximal right pulmonary artery. Two extracardiac conduit to inferior vena cava diameter ratios were created (1.0 and 1.5). A group of extracardiac conduit procedures were also created with a 5 $\mathrm{mm}$ extracardiac conduit offset toward the distal right pulmonary artery, as shown in Fig 1, $c(n=6)$.

Rate of fluid-energy dissipation. To quantify the fluiddynamic efficiency of each model, an analysis of the rate of fluid-energy dissipation was performed over a physiologic range of flow rates and conditions for each model. This analysis requires knowledge of pressure, flow, and velocity at the inlets and outlets of the right side of the heart and is especially useful because it includes all potential source of energy loss: static and kinetic energy losses caused by entrance and exit effects, and viscous dissipation losses caused by flow collision and mixing. The rate of fluid-energy dissipation analysis is simply a fluid-energy balance over the flow model. That is, the total fluid energy entering the model must equal the fluid energy leaving the model plus any incurred loss:

$$
E_{\text {loss }}=\sum E_{\text {in }}-\sum E_{\text {out }}
$$

where the total rate of fluid energy for vessel $i$ is given by:

$$
E_{i}=Q_{i}\left\{P_{i}+\frac{1}{2} \rho v_{i}^{2}\right\}
$$

for $\mathrm{i}=\mathrm{svc}$, ivc, rpa, lpa. Equation 2 represents both static and kinetic energy contributions, where $\mathrm{Q}$ is the flow rate (in cubic meters per second), $\mathrm{P}$ is the static pressure (in newtons per square meter), $v$ is the average velocity (in meters per 


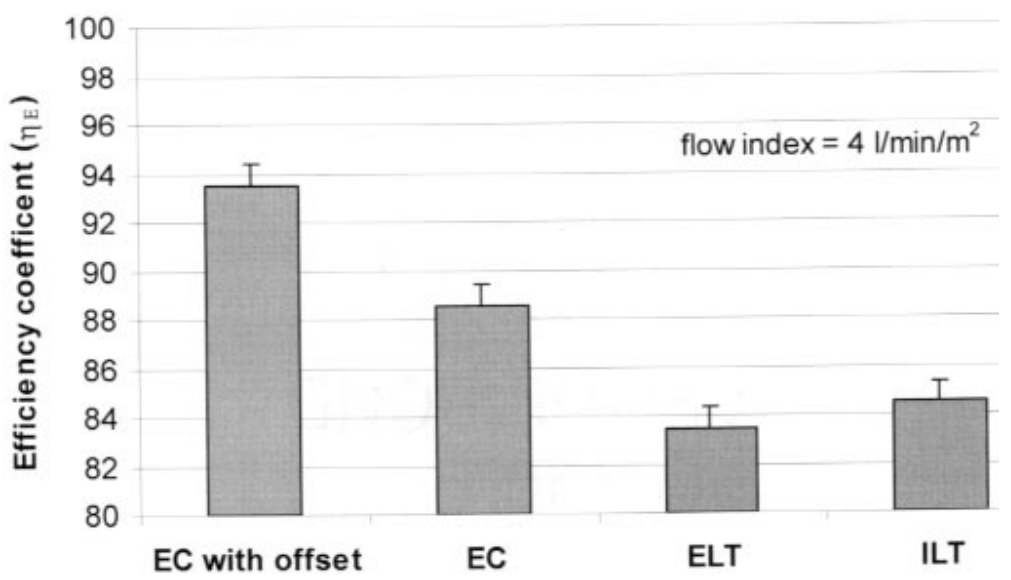

Fig 3. Overall efficiency coefficient for all configurations at a flow index of $4 \mathrm{~L} / \mathrm{min} / \mathrm{m}^{2}$. Note the EC with caval vein offset is the most efficient procedure, followed by the EC without offset (ILT, intracardiac lateral tunnel; ELT, extracardiac lateral tunnel; $E C$, extracardiac conduit).

second), and $\rho$ is the fluid density (in kilograms per cubic meter). Combining Equations 1 and 2, we arrive at an equation that represents the total energy loss occurring across each model:

$E_{l o s s}=Q_{s v c}\left\{P_{s v c}+\frac{1}{2} \rho v_{s v c}^{2}\right\}+Q_{i v c}\left\{P_{i v c}+\frac{1}{2} \rho v_{i v c}^{2}\right\}$

$-Q_{r p a}\left\{P_{r p a}+\frac{1}{2} \rho v_{r p a}^{2}\right\}-Q_{l p a}\left\{P_{l p a}+\frac{1}{2} \rho v_{l p a}^{2}\right\}(3)$

The rate of fluid-energy dissipation (power) provides useful information for comparing different geometries, but the absolute loss values cannot be readily translated into useful clinical information. To give these absolute values significance, an additional parameter was defined and referred to as the "overall efficiency coefficient" $\left(\eta_{E}\right)$ :

$\eta_{\mathrm{E}}=\left\{1-\frac{E_{\text {loss }}}{\text { Rate of total fluid energy entering the model }}\right\}$

$* 100$

which reflects the effect of flow geometry on the total rate of fluid-energy dissipation as a fraction of the total fluid energy available for fluid motion $\left(0<\eta_{\mathrm{E}}<100\right)$.

Statistical analysis. Data on the rate of fluid-energy dissipation were compared for the intracardiac and extracardiac lateral tunnel and conduit procedures for equivalent flow conditions and were expressed as the mean \pm SD of three consecutive measurements for each flow condition. Differences in the rate of fluid-energy dissipation between models and with flow index were determined by using 2-way ANOVA at a level of $P<.05$. Additionally, the effect of caval vein offset and conduit-inferior vena cava diameter ratio in the extracardiac conduit technique was determined by using a paired $t$ test.

\section{Results}

Rate of fluid-energy dissipation for the intracardiac tunnel, the extracardiac tunnel, and the extracardiac conduit is shown in Fig 2 for equivalent flow indices of 3 and $6 \mathrm{~L} / \mathrm{min} / \mathrm{m}^{2}$. Comparing the intracardiac and extracardiac tunnel procedures, the rate of fluid-energy dissipation for the extracardiac tunnel procedure was consistently higher but differences failed to reach statistical significance at each flow index $(P=.349)$. Comparison of the extracardiac conduit procedure with both tunnel procedures, however, demonstrated that the rate of fluid-energy dissipation for the extracardiac conduit was $20 \%$ less during resting output states (ie, flow rates of 2-3 L/min). At a flow index of $6 \mathrm{~L} / \mathrm{min} / \mathrm{m}^{2}$ (flow rate $=5 \mathrm{~L} / \mathrm{min}$ ), there was a marked flow-dependent increase in the rate of fluid-energy loss in each model $(P$ $\left.=1.8 \times 10^{-7}\right)$, with the extracardiac conduit being $37 \%$ more efficient compared with both tunnel procedures. In the extracardiac conduit with offset model, there was an additional $36 \%$ decrease in the rate of fluid-energy loss compared with the extracardiac conduit without offset and an overall reduction of $73 \%$ compared with the intra-atrial tunnel. The total efficiency coefficient $\eta_{\mathrm{E}}$ for all procedures studied is shown in Fig 3. The y-axis represents the ratio of the rate of fluid-energy dissipation divided by the total energy input into the model (as defined by Equation 4) for the specified model. At a flow index of $4 \mathrm{~L} / \mathrm{min} / \mathrm{m}^{2}$, the extracardiac conduit procedure with caval vein offset had the highest overall efficiency, followed by the extracardiac conduit without offset. The intra-atrial and extracardiac tunnel procedures were least efficient and comparable $(P=.564)$, with each dissipating more than $15 \%$ of the total energy 


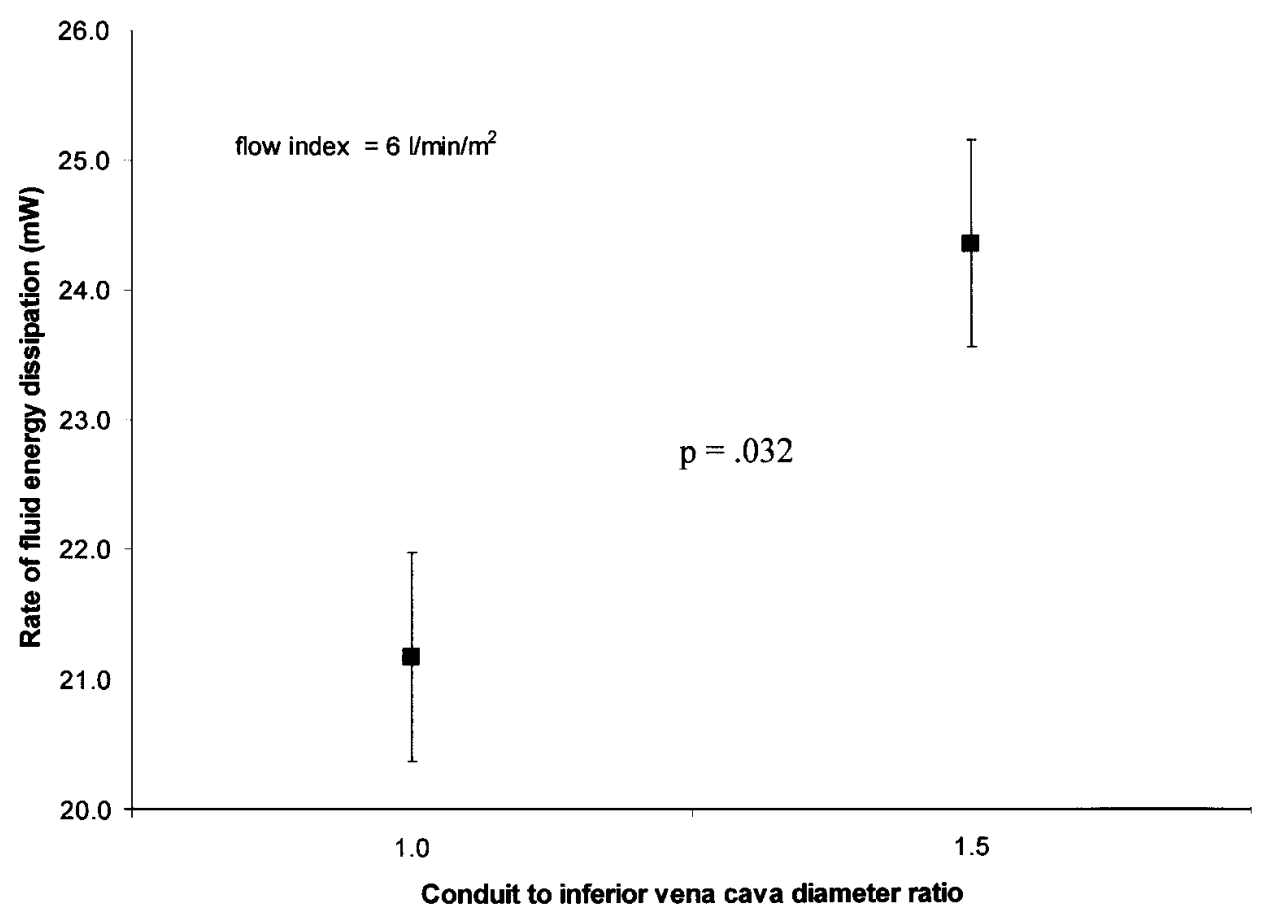

Fig 4. Effect of extracardiac conduit to inferior vena cava diameter ratio (EC/IVC) on the rate of fluid-energy dissipation.

available to drive flow across the pulmonary vasculature. At a flow index of $6 \mathrm{~L} / \mathrm{min} / \mathrm{m}^{2}$, the efficiency coefficients decreased for each model, with the tunnel models dissipating $21 \%$ of the total energy compared with $16 \%$ for the extracardiac conduit and only $10 \%$ for the extracardiac conduit procedure with caval offset. The effect of the extracardiac conduit to inferior vena cava ratio on the rate of fluid-energy dissipation is shown in Fig 4 for the extracardiac conduit procedure with no caval offset. At a flow index of $6 \mathrm{~L} / \mathrm{min} / \mathrm{m}^{2}$, the 1.5 diameter ratio had slightly higher losses compared with the diameter ratio of 1.0 representing equal inferior vena cava and extracardiac conduit diameters $(P=$ .032 ), although losses were still significantly less than those for the tunnel procedures.

\section{Discussion}

The intra-atrial TCPC proposed by de Leval and colleagues ${ }^{1}$ quickly gained popularity in the late $1980 \mathrm{~s}$ and replaced the atriopulmonary anastomosis at many centers. While there has been ample in vitro data to confirm the hemodynamic advantage of the TCPC over atriopulmonary connection, ${ }^{12-14}$ several serious complications observed in atriopulmonary connection still occur in patients with TCPC, including arrhythmias and thrombus formation. Recently, because of several theoretical and practical advantages, extracardiac procedures have been advocated for final stage conversion to the Fontan circulation. The extracardiac approach was first successfully implemented by Humes and associates ${ }^{15}$ in a patient with complex heterotaxy, and it was subsequently implemented by Marcelletti and colleagues $^{16}$ in 4 patients with complex congenital heart disease and anomalies of venous return. More recently, early and intermediate-term efficacies of this approach in a larger group of patients have been reported. ${ }^{17-19}$ To date, however, no studies have quantitatively characterized the flow dynamics of extracardiac Fontan procedures. This study compared the fluid dynamic efficiency of the intracardiac and the extracardiac total cavopulmonary procedures and offers insight into possible optimization of these procedures. Results from these experiments suggest that the extracardiac conduit procedure with caval vein offset provides the best overall performance of the TCPCs studied.

Etiology of fluid-energy losses. The improvement in the fluid dynamic efficiency of the extracardiac conduit versus the tunnel may possibly be explained by the geometry of the tunnel cross-section and the inferior vena cava to pulmonary artery connection. Short-axis B-mode echocardiographic views of the intracardiac and extracardiac tunnel procedures demonstrated irreg- 


$$
W=F m=\frac{m v_{I V C}^{2}}{2 g_{c}}\left(1-\frac{A_{I V C}}{A_{c o n d}}\right)^{2}
$$

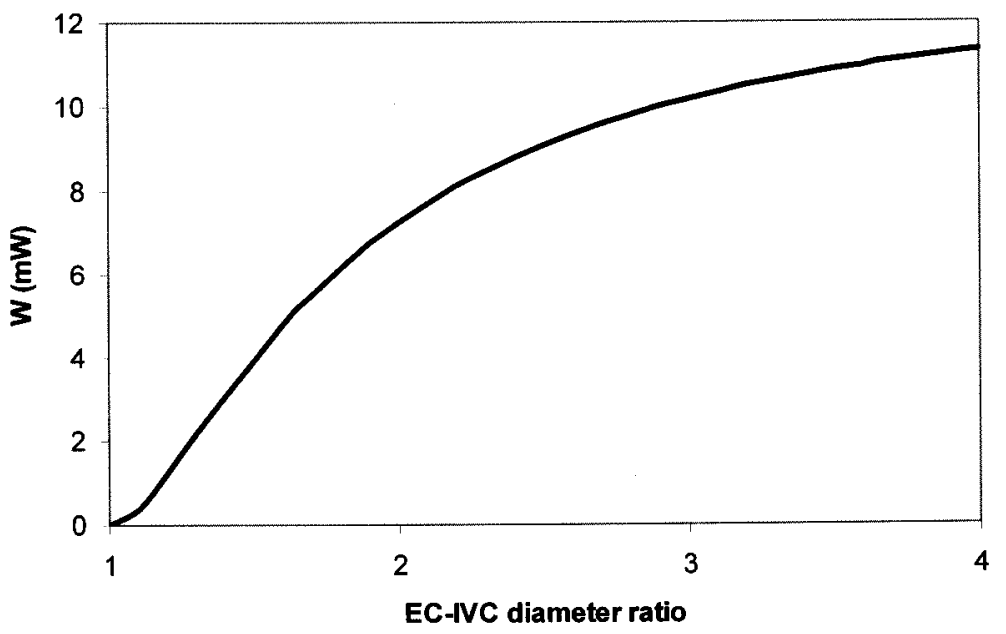

Fig 5. Effect of conduit-inferior vena cava $(E C-I V C)$ diameter ratio on the rate of fluid-energy dissipation as governed by the equation shown above. Note losses increase appreciably up to a ratio of 3.0 and then begin to reach a plateau at higher ratios. ( $W$, Rate of energy loss $(\mathrm{mW}) ; F$, friction loss $\left(\mathrm{cm}^{2} / \mathrm{s}^{2}\right) ; m$, mass flow rate $(\mathrm{g} / \mathrm{s}) ; v$, average IVC flow velocity $(\mathrm{cm} / \mathrm{s}) ; g_{c}$, gravitational constant (dimensionless); $A_{\text {cond }}$, conduit cross-sectional area $\left(\mathrm{cm}^{2}\right) ; A_{I V C}$ IVC cross-sectional area $\left(\mathrm{cm}^{2}\right)$.

ular non-circular cross-sections up to a flow index of 5 $\mathrm{L} / \mathrm{min} / \mathrm{m}^{2}$. Flow through such geometries results in asymmetric and flattened velocity profiles that are associated with increased frictional and pressure losses compared with Poiseuille flow in tubes of circular cross-section. ${ }^{20}$ As conduit flow increased further, the transmural pressure was transmitted completely to the epicardial medial side of flow area (extracardiac tunnel) and the posterior lateral wall of the right atrium (intra-atrial tunnel), thus allowing the conduit to approach a more symmetric shape radially, although longitudinal irregularity remained. An additional factor that may help explain the lower energy requirements for the conduit, compared with the extracardiac tunnel procedure, is the convex curvature of the epicardial surface of the heart. Flow through curved vessels/channels results in increased fluid-energy losses because of the development of secondary flows, separation zones, and velocity profile skewing that leads to high shear stress gradients at the outer wall. ${ }^{21}$ At the highest flow rate, the radius of curvature measured in the extracardiac tunnel tissue models would be expected to result in an additional efficiency loss of approximately $7 \%$ based on empirically derived relationships. ${ }^{20}$ Finally, in all 3 configurations, the caval veins are aligned directly across from each other (the distance between inferior and superior vena cava centerlines is zero). Caval flow stream collision and interaction results in kinetic energy losses and viscous dissipation that decreases fluid dynamic efficiency. ${ }^{7-10}$ Thus, the anatomic simplicity of achieving caval vein offset may represent a significant advantage of the extracardiac conduit technique.

In addition to the hemodynamic advantages demonstrated in these studies, the extracardiac approach has several important theoretical advantages over intra-atrial procedures, including reduced cardiopulmonary bypass time and elimination of intra-atrial suture lines, which have been shown to cause atrial dysarrhythmias in acute canine models. ${ }^{22-23}$ Table I provides a summary of the advantages and the disadvantages for the intra-atrial tunnel, extracardiac tunnel, and extracardiac conduit procedures.

Effect of conduit-inferior vena cava diameter ratio. Perhaps the most important disadvantage of the extracardiac conduit procedure that has limited its clinical use is the lack of conduit growth potential. This limitation necessitates the use of oversized conduits that may obviate some of the inherent fluid-dynamic advantages of the conduit approach. In this study, a $50 \%$ increase in the conduit to inferior vena cava diameter ratio resulted in a $13 \%$ increase in the rate of fluidenergy dissipation. This loss for the oversized conduit 
Table I. Comparison of TCPC procedures

\begin{tabular}{llll}
\hline Parameters & \multicolumn{1}{c}{ Intra-arterial tunnel } & \multicolumn{1}{c}{ Extracardiac tunnel } & Extracardiac conduit \\
\hline Aortic crossclamping & Yes & Not routinely required & Not routinely required \\
Intra-atrial suture lines & Extensive; near crista terminalis & Superficial and epicardial & No \\
Atriotomy & Yes & No & No \\
Intracardiac prosthetic material & Intra-atrial baffle & No & No \\
Thrombogenicity concern & Intra-atrial baffle material & Epicardial surface and tunnel material & Conduit material \\
Growth potential allotment & Moderate & Moderate & No \\
Late percutaneous fenestrations & Yes & Yes & No \\
Intimal peel concern & Moderate & Moderate & High \\
\hline
\end{tabular}

can be attributed to flow expansion effects at the inferior vena cava-conduit interface that cause flow separation and a loss of streamline flow though the conduit. Theoretically, such losses increase with increasing diameter ratio (Fig 5) and thus potentially impose a constraint on conduit oversizing (ie, the diameter ratio at which the inherent fluid-dynamic advantage of the conduit approach is offset by flow-expansion losses). Based on these data and empirically derived relationships governing flow-expansion processes (see Fig 6), the upper limit or critical conduit-inferior vena cava diameter ratio is approximately 2.5 . Thus, the hydrodynamic advantages of the extracardiac conduit may be best realized in older children in whom extensive conduit oversizing for growth is unnecessary.

Results from this study also underscore a rationale for the use of the extracardiac tunnel over intra-atrial procedures when concerns regarding extracardiac conduit growth preclude its use. Since there is no difference in the rate of fluid-energy dissipation between the intra-atrial and the extracardiac tunnel procedures, the extracardiac tunnel approach becomes even more compelling because the several inherent advantages of extracardiac procedures are retained without compromising the hemodynamic benefits ascribed to the basic TCPC design. Moreover, recent reports have described the use of nonprosthetic materials in the extracardiac Fontan and thus further substantiate this approach. ${ }^{24,25}$

Conversion of failing atriopulmonary connections. Besides cardiac transplantation, there are few therapeutic options for symptomatic patients with failing atriopulmonary circulations. A recently proposed option for the management of these patients, however, is lateral tunnel cavopulmonary conversion. ${ }^{19,26,27}$ The goal of this technique is to improve hemodynamic efficiency and cardiac output and reduce the occurrence of supraventricular arrhythmias and right atrial thrombus formation. However, in addition to lengthy cardiopulmonary bypass and aortic crossclamping times, which may be associated with significant mortality and morbidity in this group of very sick patients, this operation requires additional intra-atrial suture lines that may further exacerbate existing atrial conduction abnormalities. Results from these studies suggest that conversion to an extracardiac conduit may be a simpler and a more hemodynamically efficient alternative to intra-atrial lateral tunnel conversion.

Study limitations. While our in vitro experimental approach allowed us to reproduce the major physiologic parameters of the Fontan circulation, there are limitations worth noting. First, respiratory-induced alterations in pulmonary flow were not modeled. Although this is not expected to affect relative performance between procedures, it is possible that respiratory-induced pulsatility may affect absolute hydrodynamic efficiency for each model. Second, conduit velocity profiles were not measured directly. Compliant tissue models were used in these studies to reproduce the complex 3-dimensional geometry of the cavopulmonary junction that is routinely oversimplified in computational and in vitro models. The limitation of this approach, however, is that direct measurement of velocity profiles using quantitative methodology (eg, laser Doppler anemometry) is not possible because of the opaque nature of heart. Thus, we were forced to use theoretical rationale to explain the etiology of higher fluid-power losses in the tunnel compared with the conduit. Lastly, the model does not address changes in flow dynamics associated with the growth of the heart. In practice, longitudinal and radial tunnel/conduit growth is a concern for each of the procedures studied. Because models were constructed on subjects of equal body surface area and weight, the issue of growth-induced changes in tunnel/conduit and caval vein pulmonary artery junction geometry could not be quantified.

\section{Conclusions}

On the basis of these in vitro studies, we conclude that the extracardiac conduit provides improved hemodynamics over intracardiac or extracardiac lateral tun- 
nels in TCPCs. Superior vena cava-extracardiac conduit offset further enhances the hydrodynamic benefits of the extracardiac conduit, particularly at high physiologic flow rates. An extracardiac inferior vena cava-right pulmonary artery conduit may be a logical alternative to intracardiac or extracardiac lateral tunnels. This procedure is simple to perform, allows for easy caval flowstream offset, and the conduit can be moderately oversized ( $\sim 1.5$ times the inferior vena cava) without substantial hydrodynamic consequence. We believe these laboratory studies provide additional rationale for the use of extracardiac techniques for final-stage completion of TCPCs.

\section{REFERENCES}

1. de Leval MR, Kilner P, Gewillig M, Bull C, McGoon DC. Total cavopulmonary connection: a logical alternative to atriopulmonary connection for complex Fontan operations. Experimental studies and early clinical experience. J Thorac Cardiovasc Surg 1988;96:682-95.

2. Gardiner HM, Dhillon R, Bull C, de Leval MR, Deanfield JE. Prospective study of the incidence and determinants of arrhythmias after total cavopulmonary connection. Circulation 1996;94 [suppl II]:II-17-II21.

3. Pearl JM, Laks H, Stein DG, Drinkwater DC, George BL, Williams RG. Total cavopulmonary anastomosis versus conventional modified Fontan procedure. Ann Thorac Surg 1991;52: $189-96$.

4. Fishberger SB, Wernovsky G, Gentles TL, Gauvreau K, Burnett J, Mayer JE, et al. Factors that influence the development of atrial flutter after the Fontan operation. J Thorac Cardiovasc Surg 1997; 113:80-6

5. Rosenthal DN, Friedman AH, Kleinman CS, Kopf GS, Rosenfeld LE, Hellenbrand WE. Thromboembolic complications after Fontan operations. Circulation 1995;92[suppl II]:II-287-II-293.

6. Rychik J, Rome JJ, Jacobs ML. Late surgical fenestration for complications after the Fontan operation. Circulation 1997;96: 33-6.

7. Sharma S, Goudy S, Walker P, Panchal S, Ensley A, Kanter K, et al. In vitro flow experiments for the determination of optimal geometry of total cavopulmonary connection for surgical repair of children with functional single ventricle. J Am Coll Cardiol 1996;27:1264-9.

8. Dubini G, de Leval MR, Pietrabissa R, Montevecchi FM, Fumero R. A numerical fluid mechanical study of repaired congenital heart defects. Application to the total cavopulmonary connection. J Biomech 1996;26:111-21.

9. de Leval MR, Dubini G, Migliavacca F, Jalali H, Camporini G, Redington A, Pietrabissa R. Use of computational fluid dynamics in the design of surgical procedures: application to the study of competitive flows in cavopulmonary anastomoses. J Thorac Cardiovasc Surg 1996;111:502-13.

10. Lardo AC. Determination of the optimal surgical connection geometry for the repair of univentricular congenital heart disease: the Fontan procedure [dissertation]. Pittsburgh: University of Pittsburgh; 1997.
11. Lardo AC, del Nido PJ, Webber SA, Friehs I, Cape EG: Hemodynamic effect of progressive right atrial dilatation in atriopulmonary connections. J Thorac Cardiovasc Surg 1997;114:2-8.

12. Low HT, Chew YT, Lee CN. Flow studies on atriopulmonary and cavopulmonary connections of the Fontan operations for congenital heart defects. J Biomed Eng 1993;15:303-7.

13. Chew YT, Low HT, Lee CN. Flow losses in the Fontan cavopulmonary surgical connections. Proceedings of the International Society of Biomechanics. XIVth Congress, Paris, I, pp 262-3.

14. Kim YH, Walker PG, Fontaine AA, Ensley AE, Oshinski J, Sharma S, et al. Hemodynamics of the Fontan procedure: an in vitro study. J Biomed Eng 1995;117:423-8.

15. Humes RA, Feldt RH, Porter CJ, Julsrud PR, Puga FJ, Danielson GK. The modified Fontan procedure for asplenia and polysplenia syndromes. J Thorac Cardiovasc Surg 1988;92:212-8.

16. Marcelletti C, Como A, Giannico S, et al. Inferior vena cava-pulmonary artery extracardiac conduit: a new form of right heart bypass. J Thorac Cardiovasc Surg 1990;100:228-32.

17. Laschinger JC, Ringel RE, Brenner JI, McLaughlin JS. The extracardiac total cavopulmonary connection for definitive conversion to the Fontan circulation: summary of early experience and results. J Cardiac Surg 1993;8:524-33.

18. Laschinger JC, Redmond JM, Cameron DE, Kam JS, Ringel RE. Intermediate results of the extracardiac Fontan procedure. Ann Thorac Surg 1996;62:1261-7.

19. McElhinney DB, Reddy VM, Moore P, Hanley FL. Revision of previous Fontan connections to extracardiac or intra-atrial conduit cavopulmonary anastomosis. Ann Thorac Surg 1996;62:1276-82.

20. Sakiadis BC. Fluid and particle mechanics. In: Perry RH, Green DW, Maloney JO, editors. Perry's chemical engineering handbook. 6th ed. New York: McGraw-Hill Inc; 1984. p. 20-40.

21. Lefebvre XP, Pedersen EM, Hjortdal JO, Yoganathan AP. Hemodynamics. In: Lanzer P, Yoganathan AP, editors. Vascular imaging by color doppler and magnetic resonance. 1st ed. New York: Springer-Verlag; 1991. p. 58-60.

22. Gandhi SK, Bromberg BI, Rodefeld MD, Schuessler RB, Boineau JP, Cox JL, et al. Lateral tunnel suture line variation reduces atrial flutter after the modified Fontan operation. Ann Thorac Surg 1996;61:1299-309.

23. Rodefeld MD, Bromberg BI, Schuessler RB, Boineau JP, Cox JL, Huddleston CB. Atrial flutter after lateral tunnel construction in the modified Fontan operation: a canine model. J Thorac Cardiovasc Surg 1996;111:514-26.

24. van Son JA. Reddy M. Hanley FL. Extracardiac modification of the Fontan operation without use of prosthetic material. J Thorac Cardiovasc Surg 1995;110:1766-8.

25. Gundry SR, Razzouk AJ, del Rio MJ, Shirali G, Bailey LL. The optimal Fontan connection: a growing extracardiac lateral tunnel with pedicled pericardium. J Thorac Cardiovasc Surg 1997;114: 552-8.

26. Kao JM, Alejos JC, Grant PW, Williams RG, Shannon KM, Laks $\mathrm{H}$. Conversion of atriopulmonary to cavopulmonary anastomosis in the management of late arrhythmias and atrial thrombosis. Ann Thorac Surg 1994;58:1510-4.

27. Kreutzer J, Keane JF, Lock JE, Walsh EP, Jonas RA, Castaneda $\mathrm{AR}$, et al. Conversion of modified Fontan procedure to lateral tunnel cavopulmonary anastomosis. J Thoracic Cardiovasc Surg 1996;111:1169-76. 